\title{
In Conversation with Mary Jo Haddad
}

\author{
Ken Tremblay
}

A

t the helm of Canada's largest academic pediatric hospital is Mary Jo Haddad, this year's recipient of the University of Toronto's Health Policy, Management and Evaluation (HPME) and Society of Graduates Leadership Award. While Ontario's integration agenda focuses on the system, The Hospital for Sick Children (HSC, also known as SickKids) is concurrently integrating its three core businesses: patient care, research and learning for horizons that span the country. With the backdrop of HSC's vision - Healthier Children, A Better World - Mary Jo's leadership spans the globe; yet any review of milestone events at HSC bears her fingerprints, evidence of a leader focused, embraced and engaged. HQ's Ken Tremblay caught up with Mary Jo this fall.

HQ: The Society of Graduates of the University of Toronto's HPME program picked you as the 2009 recipient of their Leadership Award. What were your thoughts when you heard that news?

MH: I was truly honoured to be selected for the Leadership Award. As a graduate from the HPME program at the University of Toronto, I hold a special place in my heart for both the program and the university. Being nominated by colleagues and peers was overwhelming.

HQ: Clearly, leading a world-class organization has its challenges. How do you deal with the pressures to deliver high performance and innovation locally, nationally and on the international scene?

$\mathrm{MH}$ : It's clearly about balance, about commitment to values and ensuring that, first and foremost, you are focused on the ground. What happens in your own organization and community must be strong - for example, building on a solid history of innovation, leadership and excellence in care, research and teaching. These are fundamental to being able to share that expertise with broader constituencies across the country and across the world.

HQ: Do you ever struggle with a performance gap: seeing results elsewhere and trying to sponsor them at HSC?

$\mathrm{MH}$ : There is a common thread that weaves through every-

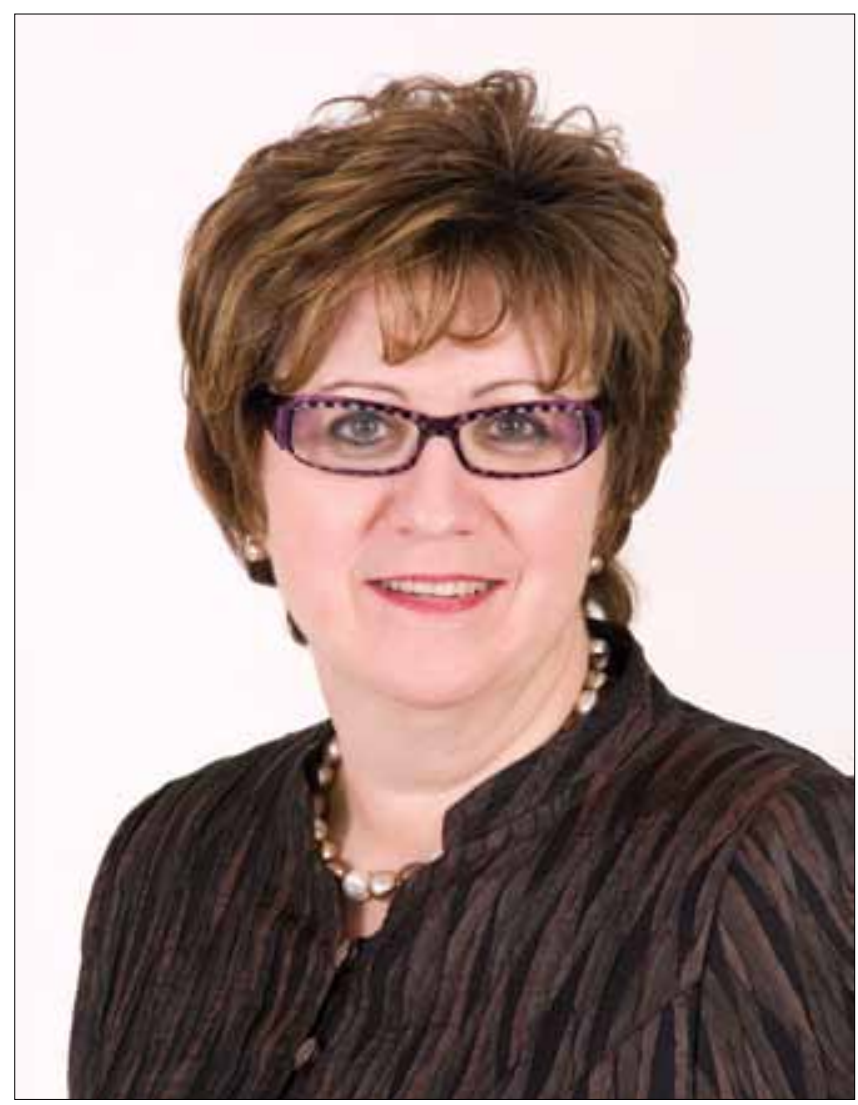

thing that you do locally, provincially, nationally and internationally. If I've learned anything in this role, it is that the world is fairly small and that we share many of the same and universally complex issues. It is very rewarding when you are able to both learn from other constituents and also share knowledge to improve children's health. That is an obvious focus of SickKids.

HQ: Given that perspective, what innovations or lessons learned do you feel hold the greatest promise for building a high-performance healthcare system in Ontario?

$\mathrm{MH}$ : We're part of a phenomenal healthcare system in both 
Canada and Ontario. An innovation that would help us on our trajectory is an electronic health system, by which we avoid the redundancies of not sharing information, reduce frustration for families from asking repetitious questions every time a provider steps into their room and increase satisfaction by helping people manoeuvre through the system. I think an e-health system will address many [improvements] if we can just move on with it.

At SickKids, we espouse the integration of care, research and teaching, and many people in other countries believe in that too. Actually making "integration" work - setting up a culture that supports research on basic questions about how one does what one does - needs an environment where you are constantly pushing the envelope, embracing new ways of thinking. Building an innovative environment brings people together around common goals, goals centred on improving care and outcomes.

HQ: What advice would you give to people in the early stages of their leadership career in healthcare?

$\mathrm{MH}$ : There are a couple of points: One is being inspired by what you do. To be a really effective leader - happy with your work and energies focused in the right areas - you need to work in a place and a system with visionary people who inspire you. The other is to be open to new learning every single day. I continue to learn in my role. I find it energizing to be around people who appreciate the leadership roles we each play, and each of us has the capacity to lead. It might not involve leading as a chief executive officer (CEO) but certainly at the bedside, in your science or in your profession, in the care you provide. Be open to those types of opportunities.

HQ: How do you reconcile the imperatives of learning and discovery with the heart-wrenching stories of courage and compassion that are so embedded in the culture of SickKids? $\mathrm{MH}$ : Each patient care experience takes us one step further in making the next patient's care even better. An environment of discovery keeps that learning at the forefront of what we do every day. There's no question that caring for critically ill children and their families or children with complex health needs who are challenged by a lifetime of issues has its challenges. Children are resilient; we can focus not only on their ailment but on their future and quality of life. You end up in a tremendously satisfying marriage, if you will, between learning, research and care. That helps us build the clinical programs we have.

HQ: As Americans wrestle with their healthcare reform, some cite the Canadian model in the negative. What lessons could the United States learn from HSC?

$\mathrm{MH}$ : The focus needs to be on care and caring in whatever system you operate: US, Canadian, European. Care and caring are at the heart of our system in Canada, as is the fundamental value of access to healthcare. There is a lot to be learned from the Canadian healthcare system. Specifically from SickKids, one of the absolute reaffirmations for me about our system is that when I compare the resources we have to do the work we do to those of a similar organization in the United States, I see we do phenomenal work. With [relatively] fewer resources, we achieve incredible results and impact. There is a lot to be learned about a focus on core values and achieving value-added benefits from every initiative or project that improves the care of patients.

HQ: You have stated that your goal is "to build an organization where communication is not an event but an ongoing iterative process that takes place at and between all levels of the organization." How do you fulfill that commitment, and how has that approach made a difference at HSC?

$\mathrm{MH}$ : We all know that communication is probably one of the largest challenges for leaders. I fundamentally believe that a commitment to transparency, a commitment to having dialogue within your organization at every level, builds greater trust and greater focus on a shared vision. I espouse to leading by example. What that means to me and my team is that we always talk about how we are going to communicate this to the organization. My belief is that we are all responsible [for effective communication]. Although we have a phenomenal communications department that facilitates the message and the methods, the responsibility for messaging and communication is from the bottom up and from the top down.

I have a forum, four to five times a year, called Dialogue with Leaders. It is attended by hundreds of people, and they can ask whatever they question they have. We get into good, sometimes very deep, dialogue about strategies, policies, new initiatives or SickKids' response to the challenges in the healthcare system or how to be a leader in the system for the changes ahead.

One of our measures at SickKids is staff engagement - that's different from satisfaction. Rather, it is engagement in the mission and vision of the organization, all the way to frontline staff. We measure staff engagement every year to assess how we can help managers when employees indicate that they are not engaged enough, that is, "I have not been asked what is important to me in my work environment or how I can contribute to the strategic vision of the organization." We have built a leadership program that not only focuses on the tools of how to manage but includes leadership: how to engage in the right types of dialogue, how to manage through conflict. It has allowed people an opportunity to come together with their peers - physician leaders, directors and front-line managers to better understand our commitment to engagement and the tools available to be more successful as leaders. We have had some phenomenal results from that [approach]. 
HQ: You mention system integration. How has that changed the clinical experience at SickKids? For example, how has your organization changed its relationships with providers outside of Toronto?

$\mathrm{MH}$ : On many fronts. A core part of our mission is "enhancing the capabilities of the child health system." What that affects is how we work day to day with the Toronto and Greater Toronto Area (GTA) partnerships we have developed. We could not do what we do without very strong pediatric and newborn supports in community hospitals across the GTA. We have some special programs with designated facilities for specialized services, like hematology or oncology clinics in communities with large populations of children who require those supports. As well, we can build standards of care in training programs that we sponsor and constantly improve.

From a system perspective, I am a big believer in healthier children, a better world; that's our vision. Healthier children means not just what happens in hospitals but what happens in terms of the way we care for our children - be it developmental support in schools or child poverty in the communities we live in and becoming engaged in that [need]. SickKids does that not only by caring for those children but by sharing information and the resources from our Research Institute.

We support integration efforts through leadership at a system level. I am the chair of the Provincial Council for Maternal Child Health, a council built over the past six years or so by a founding group of CEOs who came together and asked, if we have to do a better job for children's health in the province, how can we work better together? It became a formal council funded by the Ministry of Health and Long-Term Care. We advise the ministry on policies and investments in children and maternal child health. I have been chair for a couple of years, and it has been a tremendous move in terms of advancing a vision for Ontario's children.

HQ: Has the electronic child health network made a difference?

$\mathrm{MH}$ : Absolutely, and on a number of fronts. The soft side is that it is a tool that has enabled a conversation with every single organization in the province that cares for kids. The greatest difference is that children and parents from across the province, often referred for tertiary services, benefit from our relationships with community hospitals where those children reside. This ability to share electronically the child health record with all providers across the province is a fundamental tool for a continuity/continuum of care: tests are not repeated unnecessarily, system costs are reduced and access to care and communication are improved. That the family has access to this information has been phenomenal.
HQ: Leaders are often defined by the environment in which they function. How has University Avenue affected Mary Jo Haddad?

$\mathrm{MH}$ : It's a privilege being on University Avenue and having the exposure to tremendous leadership in each of these organizations. I get to work with outstanding colleagues. We have a tremendous relationship with the University of Toronto and certainly the Faculties of Medicine and Health Sciences. We are at the table regularly talking about training programs, education, research and policies that we can harmonize across our organizations.

University Avenue provides the opportunity to collaborate on initiatives that, for example, involve a mother with a highrisk delivery at Mount Sinai whose baby needs admission to SickKids and then stays with us for 18 years of his life because of a complex disease. Then, we transition this client to University Health Network or Sunnybrook for ongoing care and management. It is pretty amazing when you can have that type of opportunity to plan a lifetime of care. We do that better today than ever before.

HQ: When you're not at the office, what diversions help you cope with the stressors in healthcare?

MH: I have a wonderful family. I have three young children; they are my biggest diversion. My husband is incredibly supportive. I am a family lady: I love to cook and entertain, and oftentimes it's just sharing time with family and friends. When I'm not doing that, I spend a lot of time in arenas watching one of my sons play hockey. Yes, I'm a hockey mom!

\section{HQ: What would you like your leadership legacy to be at HSC?}

MH: SickKids is producing great leadership to the system locally, nationally and internationally. I am proud of that, and the organization is stronger today than it was when I became CEO.

\section{HQ: What else would you like the readers of Healthcare Quarterly to know about Mary Jo Haddad?}

$\mathrm{MH}$ : I am very fortunate to have chosen a focus and a career in children's health. That choice has been incredibly inspirational and rewarding. Throughout my career, I have worked with very talented, passionate people who inspire me. Early in my career, I worked with a nun at a Detroit hospital. She pushed me hard, which built on very strong family values of working hard and loving what you do. I have been fortunate; if I had not been part of organizations where people allowed me to grow and encouraged me to use my voice to influence policy and direction, I probably wouldn't be where I am today.

HQ: Thank you. HQ 